\title{
Postmortem findings in eight cases of influenza $\mathrm{A} / \mathrm{H} 1 \mathrm{~N} 1$
}

\author{
Daniel G Rosen ${ }^{1}$, Ana E Lopez ${ }^{2}$, Mary L Anzalone ${ }^{2}$, Dwayne A Wolf ${ }^{2}$, Sharon M Derrick ${ }^{2}$, \\ Luisa F Florez ${ }^{2}$, Morna L Gonsoulin ${ }^{2}$, Merrill O Hines III ${ }^{2}$, Roger A Mitchell², \\ Darshan R Phatak ${ }^{2}$, Kathryn Haden-Pinneri ${ }^{2}$ and Luis A Sanchez ${ }^{2}$ \\ ${ }^{1}$ Baylor College of Medicine, Houston, TX, USA and ${ }^{2}$ Harris County Medical Examiner's Office, \\ Forensic Pathology Division, Houston, TX, USA
}

\begin{abstract}
In March and early April 2009, cases of a new swine-origin influenza A (H1N1) virus were diagnosed in Mexico and the United States. Influenza virus presents as a respiratory infection with high morbidity and mortality. We describe the postmortem findings of eight confirmed cases of influenza A/H1N1 in a medical examiner setting. The eight cases falling under the jurisdiction of the Harris County Medical Examiner (Houston, TX, USA) with confirmed influenza A/H1N1 infection between June and September 2009 were included in this study. All cases were males between 6 months and 54 years of age. All adult patients had a body mass index from 31 to $49.8 \mathrm{~kg} / \mathrm{m}^{2}$. Five cases had comorbid conditions including one case with sleep apnea and mental retardation, three cases with chronic ethanolism, and one case with thymoma, sarcoidosis, and myasthenia gravis. The remaining three cases had no pre-existing medical conditions. All patients presented with severe flu-like symptoms; yet, only five were febrile. Rapid influenza diagnostic tests were performed in three cases by primary-care physicians, two of which were negative. None of the patients received antiviral medication. The average disease duration time was 8.2 days (3-14 days). A wide range of histopathological findings including tracheitis, necrotizing bronchiolitis, alveolitis, intra-alveolar hemorrhage, and hyaline membranes, both in a focal and in a diffuse distribution, were identified. Influenza A/H1N1 viral infection presents with a wide range of histological findings in a diffuse or focal distribution; most consistently with tracheitis, necrotizing bronchiolitis, and alveolitis with extensive alveolar hemorrhage. These histopathological findings at autopsy along with a clinical history of flu-like symptoms should raise suspicion for influenza A/H1N1 infection, and postmortem analysis by the reverse transcription-polymerase chain reaction (RT-PCR) is recommended for an accurate diagnosis.
\end{abstract}

Modern Pathology (2010) 23, 1449-1457; doi:10.1038/modpathol.2010.148; published online 27 August 2010

Keywords: autopsy; H1N1; histopathology; influenza A; pandemic

Influenza viruses are significant human respiratory pathogens that cause both seasonal, endemic outbreaks, and periodic unpredictable pandemics. ${ }^{1}$ Influenza A virus belong to the orthomyxoviridae family along with influenza viruses $B$ and $C$. The influenza A virion is an enveloped RNA virus of spherical to ovoid shape measuring $80-120 \mathrm{~nm}$ in diameter. New strains of the influenza A virus emerge because of a gradual process known as antigenic drift, in which mutations within the virus antibody-binding sites accumulate over time.

Correspondence: Dr DG Rosen, MD, Baylor College of Medicine, 1 Baylor Plaza, Houston, TX 77030-3411, USA.

E-mail: dgrosen@bcm.edu

Received 6 January 2010; revised 14 May 2010; accepted 17 May 2010; published online 27 August 2010
Influenza A virus is also capable of undergoing an antigenic shift that results in a new subtype of the virus. This antigenic shift causes a sudden change in antigenicity by the recombination of the influenza genome, which can occur when a cell becomes simultaneously infected by two different strains of type A influenza. The mixing of strains that can infect birds, pigs, and humans is thought to be responsible for most antigenic shifts. ${ }^{2}$

Influenza viruses are among the most common causes of human respiratory infections and among the most significant because they are associated with a high morbidity and mortality. ${ }^{2}$ In the elderly, in infants, and in people with chronic diseases, influenza is associated with especially high mortality rate. ${ }^{2}$ The worst influenza A pandemic on record in 1918 killed approximately 50 million people 
worldwide. ${ }^{1}$ In the United States, influenza results in approximately 200000 hospitalizations and 36000 deaths in a typical endemic season. Since 1700 , there have been approximately a dozen influenza A viral pandemics and in the past 120 years, pandemics occurred in 1889, 1918, 1957, and 1968. ${ }^{1}$ In March and early April 2009, cases of a new swine-origin influenza A (H1N1) virus (S-OIV) were diagnosed in Mexico and the United States. ${ }^{3,4}$ In June 2009, the World Health Organization declared an influenza pandemic. In this series, we describe the postmortem findings from eight confirmed autopsy cases of influenza A/H1N1 viral infection that were examined at the Harris County Medical Examiner's Office in Houston, TX, USA.

\section{Materials and methods}

Eight cases with confirmed influenza A/H1N1 infection autopsied at the Harris County Medical Examiner's Office between June and September 2009 were included in this study. Medical records and medical examiner investigator reports were reviewed to obtain the following clinical information: the patient's age, sex, race, social history, body weight and height, symptomatology, rapid influenza diagnostic test results, bacterial culture results, and date of initial presentation. The overall disease duration time was calculated from the date of first appearance of symptoms to the date of demise.

A detailed gross examination was performed in cases where influenza infection was suspected by clinical history. The lungs were assessed for areas of hemorrhage, congestion, and consolidation. The major bronchi and trachea were examined for erythema and exudates. Portions of lung parenchyma, hilar bronchus, and trachea were collected during autopsy and placed in $10 \%$ buffered formalin for H1N1 reverse transcription-polymerase chain reaction (RT-PCR) confirmation. ${ }^{5}$ A minimum of two tissue sections corresponding to the right and left lung were obtained for histopathological examination. In addition, a minimum of one tissue section of liver, spleen, right kidney, left kidney, left heart, right heart, and brain were also examined. A histopathological examination of the H\&E (hematoxylin-eosin)-stained slides of the lungs, trachea, and bronchi were performed in each case. Histopathology findings were semiquantitated using a fourgrade scoring system as follows: absent (A), mild $(1+)$, moderate $(2+)$, and severe $(3+)$. The quantitated parameters included: the presence of tracheal congestion and hemorrhage, necrotizing bronchitis, necrotizing bronchiolitis, necrotizing alveolitis, alveolar edema, intra-alveolar hemorrhage, hyaline membranes, lymphocytes and macrophage infiltration, acute inflammation, eosinophils, and capillary thrombosis. H\&E-stained slides from all eight autopsies were available for examination.
A total of 22 suspected cases were tested for H1N1 RNA by RT-PCR between June and September 2009. Eight of these cases (36\%) tested positive for the H1N1 virus. Of the eight positive cases, six cases were tested at the Centers for Disease Control and Prevention (CDC, Atlanta, GA, USA) and two cases were tested at the City of Houston Department of Health and Human Services Laboratory. Immunohistochemical stain was performed by the CDC on the six cases submitted for H1N1 testing according to their protocol. No immunohistochemical demonstration was performed on the remaining two cases.

\section{Results}

\section{Clinical Characteristics}

A summary of the clinical characteristics of the positive H1N1 cases is shown in Table 1. All cases were male, between 6 months and 54 years of age (median $=27$ years). Four were Hispanic, two were Black, and two were Caucasian. All of the adult decedents were obese, with body mass indices from 31 to $49.8 \mathrm{~kg} / \mathrm{m}^{2}$. One decedent had a past medical history of mental retardation and sleep apnea, and another had a past medical history of sarcoidosis, myasthenia gravis, and thymoma. Three decedents had a history of chronic ethanolism. The remaining cases had no relevant medical history. All decedents presented with severe flu-like symptoms; yet, only five were febrile $\left(>99^{\circ} \mathrm{F}\right)$. Rapid influenza diagnostic tests were performed in three cases by primarycare physicians, two of which were negative. None of the decedents received antiviral medication. Postmortem lung and blood bacterial cultures performed at autopsy revealed bacterial growth in five cases (Table 1). Bacterial cultures were polymicrobial in four cases and monomicrobial in one case. The average disease duration time after the onset of symptoms was 8.2 days, with a disease duration time ranging from 3 to 14 days.

\section{Autopsy Findings}

The external examination of all cases was unremarkable with the exception of obesity findings. On internal examination, all decedents had extensive mucosal erythema and edema of the larynx, trachea, and bronchi (Figure 1). Focal mucosal hemorrhage was noted in some cases (Figure 1a). In most cases the lungs were diffusely congested and hemorrhagic (Figure 2). A summary of the histological findings is shown in Table 2. Microscopic examination demonstrated diffuse lymphocytes and macrophages infiltrating the larynx and mainstem bronchi. The amount of lymphocytic and macrophage infiltrate was variable, but diffuse along the mucosa of the upper airway in all cases. Reactive epithelial hyperplasia was common in areas where the inflammation was more prominent (Figure 3a). 


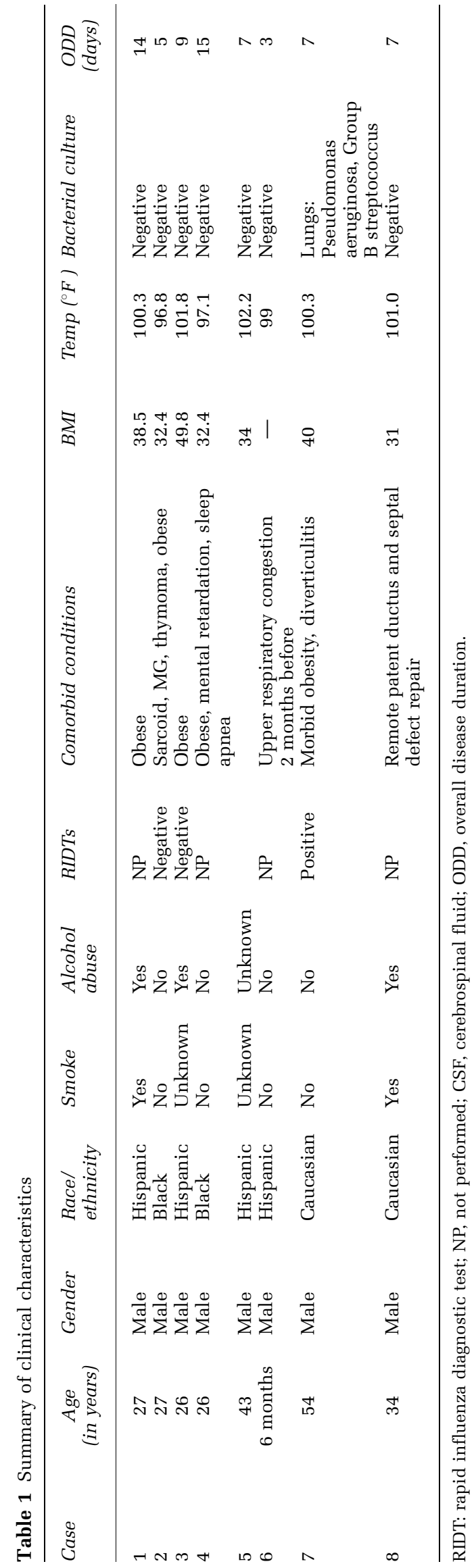

A broad spectrum of bronchiolar and alveolar lesions, including various degrees of necrosis of the bronchiolar wall with desquamation of the epithelium, submucosal edema, and vascular congestion (Figure 3b), was shown. Hyaline membranes lining alveolar ducts and adjacent alveoli were found in all cases (Figure 3c and d). Alveolar hemorrhage (Figure 3e and f) was focal in two cases and diffuse in six cases. The alveolar air spaces contained edema fluid, strands of fibrin, desquamated epithelial cells, erythrocytes, and inflammatory cells composed mostly of lymphocytes with occasional neutrophils and eosinophils (Figure 3g). Lung sections of the decedent with a history of sarcoidosis showed diffuse and extensive areas of non-caseating granulomas with giant cells, consistent with sarcoidosis. Patchy areas showing diffuse alveolar damage with intra-alveolar hemorrhage and necrotizing bronchiolitis were also present in this decedent. Apart from the case of sarcoidosis, another case (case 4) showed rare giant cells in the lung sections (Figure 3h). (There was no history of intravenous drug abuse, sarcoidosis, tuberculosis, or fungal infection in this particular case.)

Immunohistochemical stains for H1N1 were performed on lung tissue in six cases at the CDC. Three cases were reported as positive in alveolar-lining pneumocytes (not shown), and three cases were reported as negative.

\section{Extrapulmonary Findings}

Hepatomegaly with moderate-to-severe micro- and macrovesicular steatosis was seen in five decedents, two of which corresponded to the cases with a social history of chronic alcoholism. Cardiomegaly was a feature in five decedents. One decedent had arterionephrosclerosis. Cerebral edema was present in one decedent.

\section{Discussion}

In this series we describe the postmortem findings in eight confirmed deaths due to influenza A/H1N1 viral infection presenting to the Harris County Medical Examiner's Office in Houston, TX, USA. We found that influenza A/H1N1 has a wide range of histological findings in the lungs, in a diffuse or focal distribution. Most consistently, we found tracheitis, necrotizing bronchiolitis, and alveolitis with extensive hemorrhage.

Secondary bacterial infection has been reported in 20 to $38 \%$ of the cases in the 2009 influenza pandemic. $^{6-8}$ The most common bacteria involved are Staphylococcus aureus, Streptococcus pneumoniae, Streptococcus pyogenes, Streptococcus mitis, and Haemophilus influenza. ${ }^{9}$ We found that four of the eight cases had polymicrobial culture growth and one had monobacterial culture growth. However, histopathological evidence of typical 

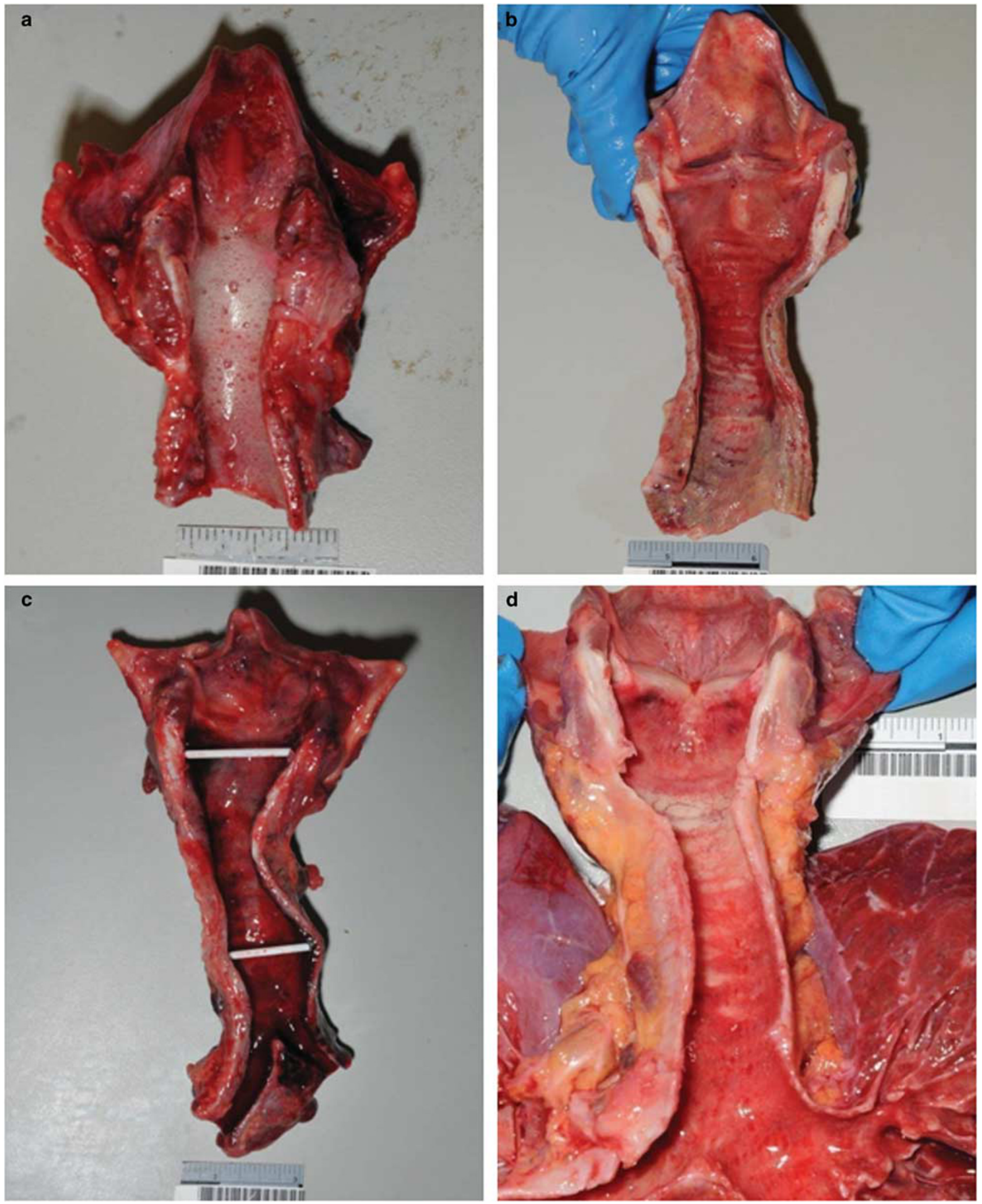

Figure 1 Representative examples of laryngeal gross specimens. The specimens shown correspond to (a) case 1, (b) case 2, (c) case 3, and (d) case 4. Marked hyperemia, mucosal hemorrhages, and edema of the larynx, trachea, and main bronchi are present.

superimposed bacterial pneumonia was observed in only one case where Pseudomonas aeruginosa, Group B streptococcus and Streptococcus constellatus were isolated. In contrast to recently published studies, we found a much lower rate of bacterial coinfection. Our cohort consists of patients who did 

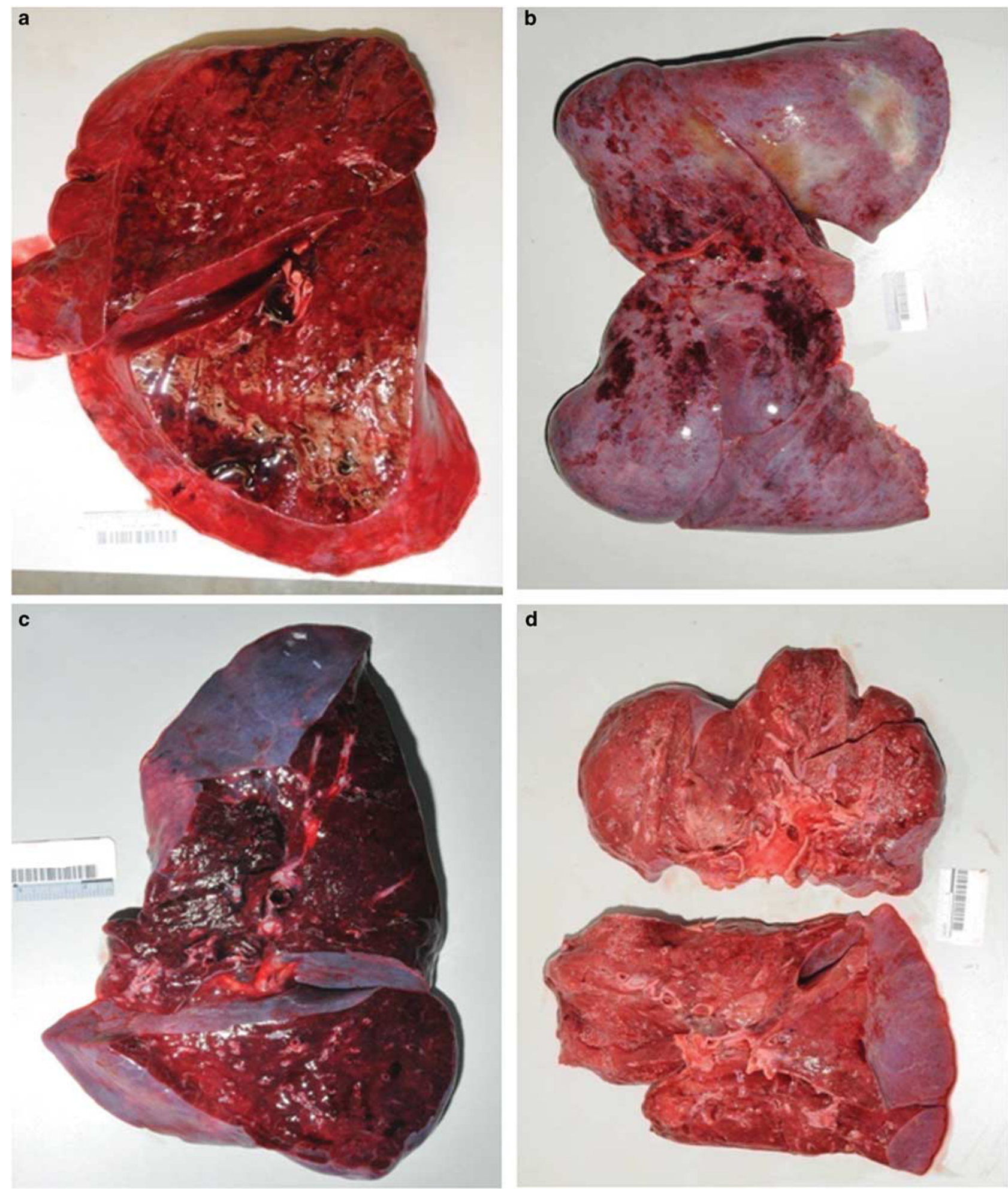

Figure 2 Representative examples of lung gross specimens. The specimens shown correspond to (a) cases 1, (b) case 2, (c) case 3, and (d) case 4 . The lungs showed a spectrum of gross findings, which included diffuse congestion (a), pleural hemorrhages (b), hemorrhagic consolidation and diffuse congestion (c), and subtle, mild congestion (d).

not receive any type of antiviral medication or prolonged hospitalization, which may account for the difference in bacterial coinfection rates.
The presence of predisposing conditions among patients who present with severe disease varies widely among reports. ${ }^{6,8,10}$ Nearly $25 \%$ of ICU 


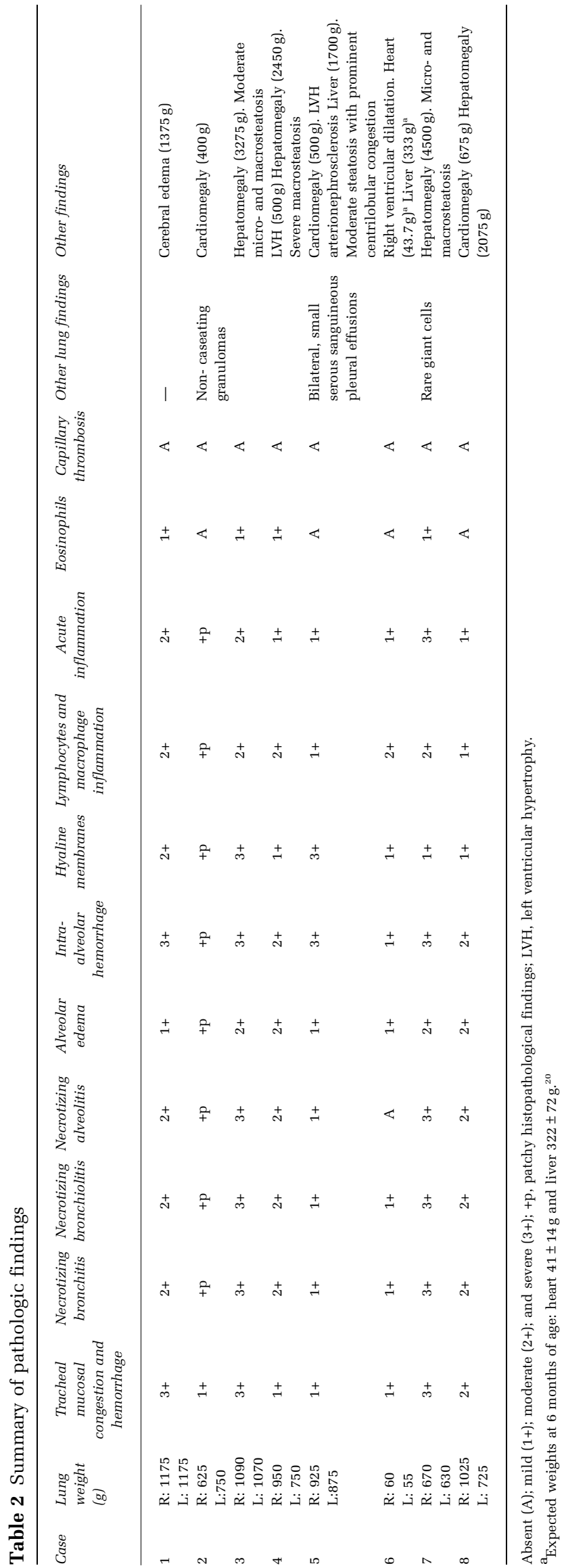

patients in Australia and New Zealand had predisposing conditions, along with $98 \%$ of cases in Canada, $84 \%$ in Mexico, and $87 \%$ in Spain. ${ }^{6,8,10,11}$ In our series, all seven adult decedents were obese with body mass indices $>31 \mathrm{~kg} / \mathrm{m}^{2}$. In addition, one decedent had mental retardation and sleep apnea, and another had sarcoidosis, myasthenia gravis, and thymoma. Obesity has been reported in $28-36 \%$ of critically ill patients with influenza A/H1N1. ${ }^{6,8,10,11}$ Further studies are needed to determine how these comorbidities may contribute to the severity and outcome of the disease.

The first description of findings in H1N1 infection are from the 1918 influenza pandemic: diffuse alveolar damage (DAD) with hyaline membranes, necrosis of the alveolar epithelium, small vessel thrombosis, and intra-alveolar hemorrhage. ${ }^{12}$ During the current pandemic, Mauad et $a l^{7}$ describe three distinct patterns of lung involvement in $\mathrm{H} 1 \mathrm{~N} 1$ infection: classic exudative DAD, severe necrotizing bronchiolitis, and exudative DAD with an intense hemorrhagic component. The researchers describe an association between necrotizing bronchiolitis and the presence of a bacterial coinfection. An association is also noted between severe alveolar hemorrhage and comorbidities, such as chronic cardiovascular disease and coagulopathies. This is in contrast to our findings where all of the cases had some degree of necrotizing bronchiolitis, yet only one case had an associated bacterial coinfection. In addition, all of our cases had alveolar hemorrhage; however, the only comorbid conditions in our cases were chronic ethanolism in three decedents, and sarcoidosis, myasthenia gravis, and thymoma in another decedent. Studies performed in guinea pigs showed that a neutrophilic infiltrate can be present in the very early stages of H1N1 infection causing acute bronchiolitis, bronchointerstitial pneumonia, and alveolitis. ${ }^{13}$ However, other studies found that an outstanding feature in the early stage of infection is the absence of neutrophils in the infiltrate. ${ }^{12}$ Even though it may be convenient to correlate the clinical findings with the histopathological findings, it remains unclear how the histopathological findings in H1N1 infection truly correlate with clinical comorbidities and bacterial coinfection because tissue sampling, patient susceptibilities, disease onset, and grading and interpretation of the histopathological findings are variable. It is important to note that histological findings on autopsy specimens represent the most severe cases.

Only a few studies with a limited number of cases have been published describing the histopathological findings of seasonal influenza in humans. ${ }^{14,15}$ In uncomplicated human influenza virus infection with predominant viral attachment to the tracheal and bronchial epithelial cells, there is a transient tracheo-bronchitis. Compared with healthy controls, patients with influenza infection show a mild increase in lymphocytic inflammation of the interalveolar septa and thickened surface epithelium. 
Diffuse necrotizing tracheobronchitis, edema, hyperemia, and lymphohistiocytic infiltrate of the lamina propria can be readily visible from day $1 .^{14,15}$ Autopsy studies have shown that the histopathological changes in the lung in complicated influenza viral pneumonia with extension of viral infection into the alveoli consist of diffuse alveolar damage characterized by desquamation of alveolar epithelium, intra-alveolar edema and hemorrhage, hyaline membranes, and capillary thrombi and congestion. Neutrophils and eosinophils may be present. In addition, bronchioles may show necrotizing bronchiolitis. ${ }^{14}$

Influenza A virus targets both the upper respiratory epithelium and alveolar epithelium; ${ }^{16}$ however, the infection does not cause a pathognomonic inflammatory reaction. ${ }^{17}$ Biopsy findings in patients with influenza A/H1N1 infection lack a characteristic pattern. Findings consist of mild-to-moderate $\mathrm{DAD}$, focal acute inflammation, or a pattern resem- bling bronchiolitis obliterans organizing pneumonia (BOOP). ${ }^{18}$ Severe cases are categorized by extensive necrotizing tracheobronchitis, with ulceration and sloughing of the bronchial mucosa. ${ }^{12}$ Larger studies are needed to determine the true nature of these findings.

Extrapulmonary findings were observed by some researchers in the setting of influenza H1N1 viral infection including renal acute tubular necrosis, centrilobular necrosis of the liver, and hemorrhagic necrosis of the adrenal glands. Of note, erythrophagocytosis in lymph nodes, spleen, bone marrow, and the liver have also been described. ${ }^{7,17}$ None of these findings were seen in our cases; however, the cases that we examined had no prolonged hospitalization, which may account for these extrapulmonary changes. Compared with the previous studies, our series have an increased number of cases with moderate-to-severe hepatic steatosis (five of the seven adult cases) and cardiomegaly (five of seven
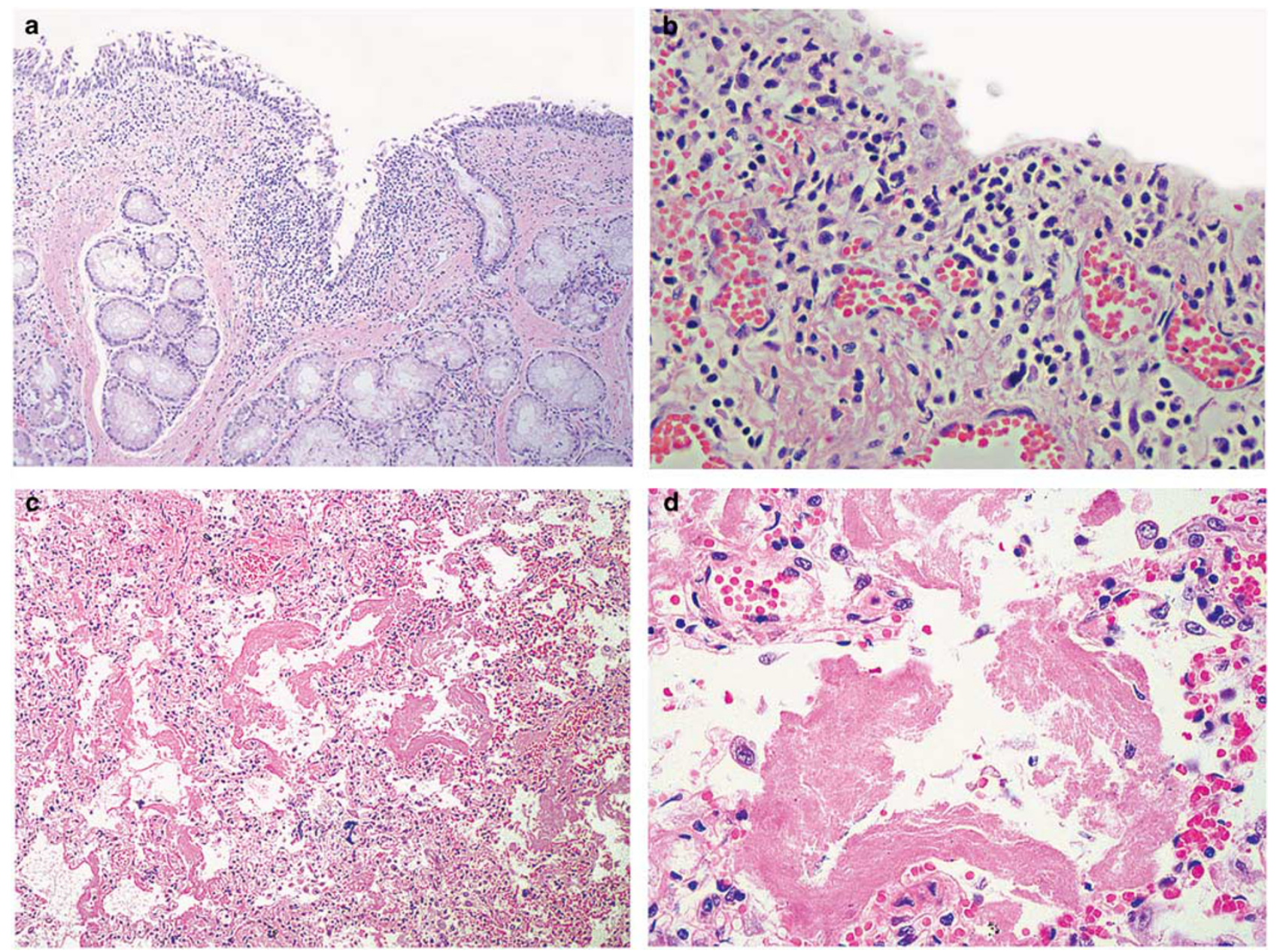

Figure 3 Representative examples of microscopic findings in the lung section. Lymphocytes and macrophage infiltrate along the mucosa of the trachea (a, hematoxylin-eosin (H\&E), $\times 4$ ). Necrosis of the bronchiolar wall with lymphocyte and macrophage infiltrate, submucosal edema, and vascular congestion (b, H\&E, $\times 20)$. Hyaline membranes lining an alveolar duct and adjacent alveoli $(\mathbf{c}, \mathbf{d}, \mathrm{H} \& \mathrm{E}$, $\times 4$ and $\times 20$ ). Areas of alveolar hemorrhage $(\mathbf{e}, \mathbf{f}, \mathrm{H} \& \mathrm{E}, \times 4)$. The alveolar air spaces have edema fluid, strands of fibrin, prominent hyaline membranes, desquamated epithelial cells, erythrocytes, and inflammatory cells mostly composed of lymphocytes with occasional neutrophils and eosinophils $(\mathrm{g}, \mathrm{H} \& \mathrm{E}, \times 20)$. Rare giant cells (arrow heads, h, H\&E, $\times 4$ ). 

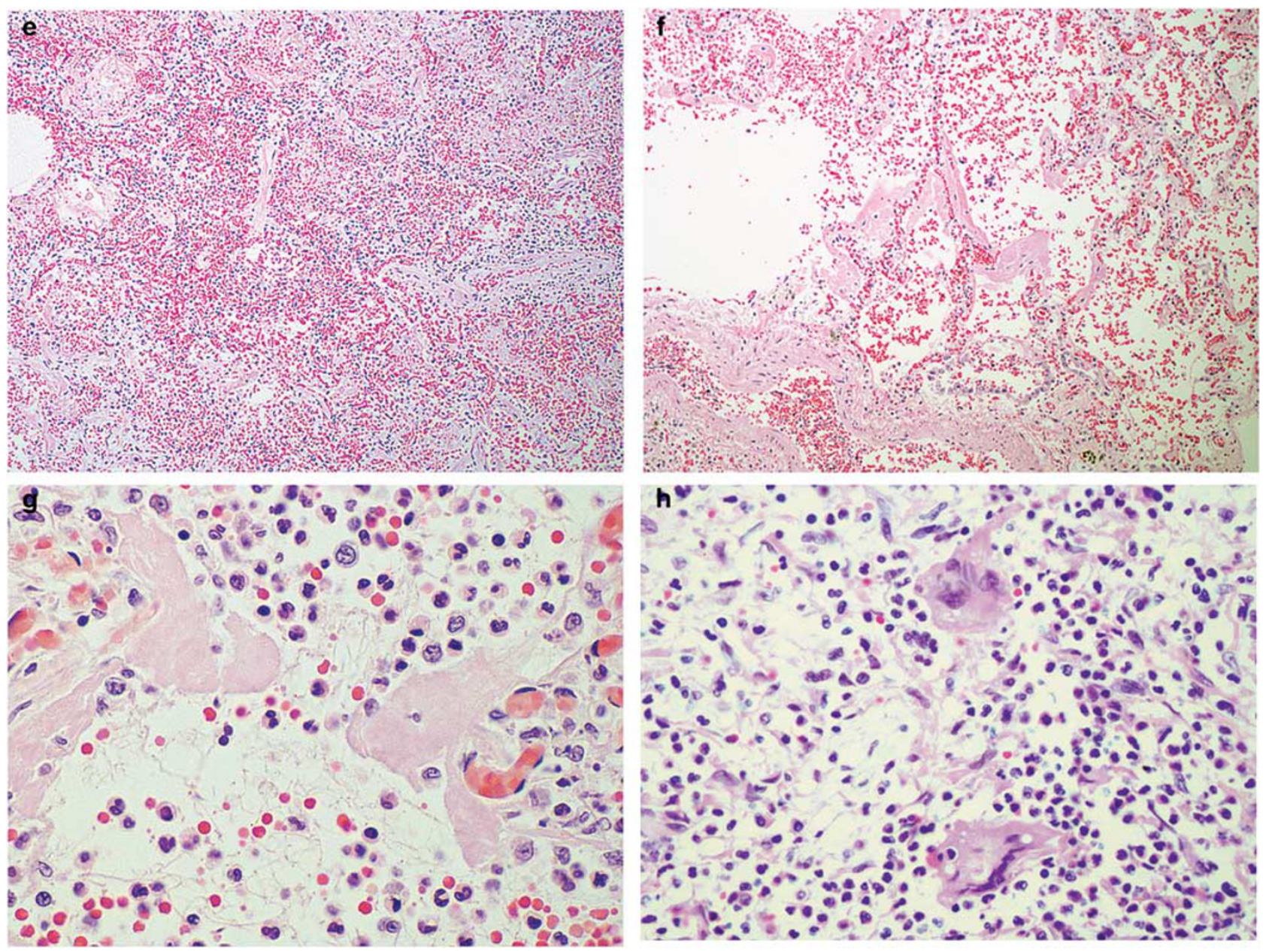

Figure 3 Continued.

cases), likely representing comorbid conditions such as chronic ethanolism and hypertension. In addition, all of our adult decedents were obese (BMI $>30$ ), which correlates with the findings of another recently published series where obesity was noted in $72 \%$ of adult and adolescent cases of $\mathrm{H} 1 \mathrm{~N} 1 \mathrm{viral}$ infection. ${ }^{19}$

\section{Conclusions}

Because of the nature of the cases falling under the jurisdiction of the medical examiner, these eight autopsy cases are most representative of the natural progression of this disease, in that none of these cases received antiviral medications or had prolonged hospitalizations. H1N1 influenza viral infection presents with a constellation of histopathological findings in a diffuse or focal distribution; most consistently with tracheitis, necrotizing bronchiolitis, and alveolitis with extensive intraalveolar hemorrhage. Encountering these histopathological findings should raise a high level of suspicion, and postmortem analysis by RT-PCR should be performed to rule out influenza A/H1N1 infection.

\section{Disclosure/conflict of interest}

The authors declare no conflict of interest.

\section{References}

1 Zimmer SM, Burke DS. Historical perspective-emergence of influenza A (H1N1) viruses. N Engl J Med 2009;361:279-285.

2 Cohen J, Powderly WG. Infectious Diseases. 2nd edn. Mosby: Edinburgh; New York, 2004.

3 Centers for Disease Control and Prevention. Update: swine influenza A (H1N1) infections-California and Texas, April 2009. MMWR Morb Mortal Wkly Rep (2009);58:435-437.

4 Centers for Disease Control and Prevention. Update: infections with a swine-origin influenza A (H1N1) virus-United States and other countries, April 28, 2009. MMWR Morb Mortal Wkly Rep 2009;58: $431-433$. 
5 Centers for Disease Control and Prevention, Coordinating Center for Infectious Diseases. Guidelines for the Submission of Tissue Specimens for the Pathologic Evaluation of Influenza Virus Infections 2010; Available from: http:// www.cdc.gov/h1n1flu/tissuesubmission.htm.

6 Kumar A, Zarychanski R, Pinto R, et al. Critically ill patients with 2009 influenza A(H1N1) infection in Canada. JAMA 2009;302:1872-1879.

7 Mauad T, Hajjar LA, Callegari GD, et al. Lung pathology in fatal novel human influenza A (H1N1) infection. Am J Respir Crit Care Med 2010;181:72-79.

8 Webb SA, Pettila V, Seppelt I, et al. Critical care services and $2009 \mathrm{H} 1 \mathrm{~N} 1$ influenza in Australia and New Zealand. N Engl J Med 2009;361:1925-1934.

9 Louie J, Jean C, Chen T-H, et al. Bacterial coinfections in lung tissue specimens from fatal cases of 2009 pandemic influenza A (H1N1)-United States, MayAugust 2009. MMWR Morb Mortal Wkly Rep 2009;58:1071-1074.

10 Dominguez-Cherit G, Lapinsky SE, Macias AE, et al. Critically ill patients with 2009 influenza A (H1N1) in Mexico. JAMA 2009;302:1880-1887.

11 Rello J, Rodriguez A, Ibanez P, et al. Intensive care adult patients with severe respiratory failure caused by Influenza A (H1N1)v in Spain. Crit Care 2009;13:R148.

12 Taubenberger JK, Morens DM. The pathology of influenza virus infections. Annu Rev Pathol 2008;3:499-522.
13 Tang X, Chong KT. Histopathology and growth kinetics of influenza viruses (H1N1 and H3N2) in the upper and lower airways of guinea pigs. J Gen Virol 2009;90:386-391.

14 Camner P, Jarstrand C, Philipson K. Tracheobronchial clearance in patients with influenza. Am Rev Respir Dis 1973;108:131-135.

15 Levandowski RA, Gerrity TR, Garrard CS. Modifications of lung clearance mechanisms by acute influenza A infection. J Lab Clin Med 1985;106:428-432.

16 van Riel D, Munster VJ, de Wit E, et al. Human and avian influenza viruses target different cells in the lower respiratory tract of humans and other mammals. Am J Pathol 2007;171:1215-1223.

17 Soto-Abraham MV, Soriano-Rosas J, Diaz-Quinonez A, et al. Pathological changes associated with the 2009 H1N1 virus. N Engl J Med 2009;361:2001-2003.

18 Yeldandi AV, Colby TV. Pathologic features of lung biopsy specimens from influenza pneumonia cases. Hum Pathol 1994;25:47-53.

19 Gill JR, Sheng ZM, Ely SF, et al. Pulmonary pathologic findings of fatal 2009 pandemic influenza A/H1N1 viral infections. Arch Pathol Lab Med 2010;134: 235-243.

20 Thompson WS, Cohle SD. Fifteen-year retrospective study of infant organ weights and revision of standard weight tables. J Forensic Sci 2004;49:575-585. 